\title{
Formalin-Fixed Paraffin-Embedded RNA
}

National Cancer Institute

\section{Source}

National Cancer Institute. Formalin-Fixed Paraffin-Embedded RNA. NCI Thesaurus. Code C156436.

An RNA sample derived from a formalin-fixed paraffin-embedded tissue sample. 